\title{
1 GWAS in the southern African context
}

2 Yolandi Swart ${ }^{1 \uparrow^{*}}$, Gerald van Eeden ${ }^{1 \uparrow}$, Caitlin Uren ${ }^{1,2}$, Gian van der Spuy ${ }^{1,3}$, Gerard Tromp ${ }^{1,2,3}$,

3 Marlo Möller ${ }^{1,2 *}$

$4 \quad{ }^{1}$ DSI-NRF Centre of Excellence for Biomedical Tuberculosis Research, South African Medical

5 Research Council Centre for Tuberculosis Research, Division of Molecular Biology and

6 Human Genetics, Faculty of Medicine and Health Sciences, Stellenbosch University, Cape

7 Town, South Africa

$8{ }^{2}$ Centre for Bioinformatics and Computational Biology, Stellenbosch University, 7602

9 Stellenbosch, South Africa

$10{ }^{3}$ SAMRC-SHIP South African Tuberculosis Bioinformatics Initiative (SATBBI), Center for

11 Bioinformatics and Computational Biology

12

$13 *$ Corresponding authors

14 E-mail: marlom@sun.ac.za

yolandi01@sun.ac.za

17 These authors contributed equally to this work. 


\section{Abstract}

25 Researchers would generally adjust for the possible confounding effect of population structure

26 by considering global ancestry proportions or top principle components. Alternatively, researchers would conduct admixture mapping to increase the power to detect variants with an ancestry effect. This is sufficient in simple admixture scenarios, however, populations from southern Africa can be complex multi-way admixed populations. Duan et al. (2018) first described local ancestry adjusted allelic (LAAA) analysis as a robust method for discovering association signals, while producing minimal false-positives. Their simulation study, however, was limited to a two-way admixed population. Realizing that their findings might not translate to other admixture scenarios, we simulated a three- and five-way admixed population to compare the LAAA model to other models commonly used in GWAS. We found that, given our admixture scenarios, the LAAA model identifies the most causal variants in most of the phenotypes we tested across both the three-way and five-way admixed populations. The LAAA model also produced a high number of false-positives which was potentially caused by the ancestry effect size that we assumed. Considering the extent to which the various models tested differed in their results and considering that the source of a given association is unknown, we recommend that researchers use multiple GWAS models when analysing populations with complex ancestry. 


\section{Introduction}

Differential assortment of ancestral allele frequencies across a genome results from selection, mutation or genetic drift, when previously isolated populations interbreed $(1,2)$. Taking advantage of these differences in allele frequencies can help identify population-specific disease risk alleles associated with disease phenotypes due to various ancestries being exposed to distinct environments and pathogens (3). Admixed populations present unique opportunities to identify ancestry-specific disease risk alleles for various populations simultaneously. African ancestries harbour the most genetic diversity and contain more complex LD blocks than other continental populations and the precise evolutionary events that shaped their genomes are mostly unknown (4,5). When the historical events are unknown, it is often difficult to know beforehand which effect (allele, ancestry or the interaction between them) has the most significant effect on the disease phenotype under study. Therefore, studying admixed African populations presents a rich opportunity for the discovery of ancestry-specific disease risk alleles, however, the complex genomic architecture of admixed African genomes warrants careful consideration of both global and local ancestry.

A genome-wide association study (GWAS) is commonly used to discover associations between single nucleotide polymorphisms (SNPs) and a diverse spectrum of complex traits of interest (6). Attributing associations at the SNP level, however, requires large sample sizes when the SNP being tested has a small effect on the trait of interest. GWAS is further complicated when individuals have admixture-induced linkage disequilibrium (admixture LD) blocks. Admixture mapping uses the admixture LD blocks inherited from a specific ancestral population to test for an association with the trait of interest (2). Hence, the ancestry rather than the genotypes are traced in the association process (7) and the SNPs that affect the trait of interest can only be localised to their respective ancestral blocks (presented as an admixture peak on a Manhattan plot). There are also smaller LD blocks within admixture LD blocks that originated prior to admixture that are inherited from the parent populations that contributed to the admixture (ancestry LD). Admixture LD and ancestry LD cause population stratification in a GWAS which reduces the power to detect a significant association and could lead to increased falsepositives and false-negatives (8). Therefore, at a given locus within an admixed population there will be groups of individuals with similar LD. If one or more of these groups is disproportionately represented within a given phenotype, a false association will exist between 
that group and that phenotype. Many approaches address this problem by including global ancestry and local ancestry in GWAS models (9-11).

Duan et al. (8) expanded on these approaches by additionally modelling the interaction between allelic effects and ancestry effects. They proposed a two-step approach, called the Local Ancestry Adjusted Allelic (LAAA) testing procedure, that first detects associations by jointly modelling allelic effects, ancestry effects and interaction effects and then determines the source of the association. Their approach proved robust and powerful, but it was only assessed in a two-way admixed population. Two-way admixed populations generally have simple demographic histories and often originate from single pulse admixture events. Therefore, it remains to be determined if this model will be able to capture the correct association signals with minimal power loss and spurious associations (including both false-positives and falsenegatives) in complex multi-way admixed populations. We believe that testing this will go a long way to identifying robust models that can be used in a wide array of demographic scenarios.

We used the complex demographic history of the Nama and the South African Coloured (SAC) populations to simulate a three-way admixed population (as represented by the Nama) and a five-way admixed population (as represented by the SAC). The genotypes from these simulated populations were used to simulate three phenotypes, each with a different association signal, to determine if the LAAA model will be able to robustly capture the true causal variants regardless of the source of the association or the complexity of the admixture. We also compare other models commonly used in GWAS and we investigate the efficacy of each model when presented with true versus inferred ancestry.

\section{Material and Methods}

\section{Simulating Genotypes}

msprime (12) was used to jointly simulate the demographic history (Figure 1) of the Nama, South African Coloured (SAC), Han Chinese in Beijing, China (CHB), British From England and Scotland (GBR), Gujarati Indians in Houston, Texas, USA (GIH), Gumuz, Luhya in Webuye, Kenya (LWK) and Mende in Sierra Leone (MSL). The model describing the demographic history was defined in a Demes Specification (https://github.com/popsimconsortium/demes-python) that was then converted to a msprime demographic model using the 
demes python library. Demes Specifications are YAML files that contain information about populations, their properties (like effective population size changes) and the relationship of a population to other populations.

The demographic history of the Nama, an indigenous population of southern Africa (13) that forms part of a larger group of geographically close and culturally related individuals known collectively as the "Khoe-San", is complex and extends back to the emergence of modern humans. The Khoe-San are reported to have the most divergent lineages compared to other living populations (14-18) and it is believed that they have largely remained isolated until $\sim 2000$ years ago $(13,14,19)$. The Nama is reported to have experienced 5-25\% gene flow from eastern African caprid and cattle pastoralists 2000 years ago (20). These events were finally followed by recent admixture with European colonists $\sim 250$ years ago. The demographic model for the Nama (Fig 1) was based on the above mentioned events and a conference poster by Ragsdale et al. (21) where they inferred detailed parameterized demographic models for five present day populations (GBR, Gumuz, LWK, MSL and Nama) using joint allele frequency and LD statistics. The Khoe-San also contribute a significant ancestral component to a South African population that represents a highly admixed group from multiple ancestral populations (SAC) (22)(23). Many non-African and African populations moved into southern Africa over the last $\sim 600$ years and integrated with the Khoe-San (22). Uren et al. (13) found that the SAC has an estimated 32-43\% Khoe-San, 20-36\% Bantu-speaking African, 21-28\% European and 9-11\% East and South-East Asian ancestral contribution. The demographic model for the SAC (Fig 1) was based on the above ancestral proportions with a single admixture event, for simplicity, between the ancestral populations $\sim 400$ years ago.

Fig 1. The demographic histories of the simulated populations. Thick solid lines with open arrowheads indicate an ancestor $->$ descendant relation, dashed lines indicate an admixture pulse and faint solid lines with closed arrowheads indicate continuous migration.

The demographic models were used to simulate DNA sequence evolution of 5000 Nama and 5000 SAC individuals as tree sequences (Fig 2). We assumed a generation time of 25 years, a mutation rate of $1.29 \mathrm{e}-08$ and a recombination rate as specified by the Combined HapMap II recombination map. The CHB, GBR, GIH, LWK and MSL (1000 individuals of each) were simulated to act as reference populations for the Nama and the SAC. The Nama acted as a reference population for any Khoe-San ancestral contribution in the Nama and the SAC. Our 
aim with this simulation was not to create models that accurately represent the demographic history of the Nama or the SAC, but to simulate realistic genotype data for a three-way and five-way admixed population as represented by the Nama and the SAC. The tree sequence was then used to create VCFs using tskit (24). The number of SNPs in the resultant VCFs was reduced to $\sim 400000$ SNPs to reduce model runtime by randomly selecting variants. We also wrote a custom script that works its way up the tree sequence to find the ancestry at a given locus at a given time using the migration history of that locus, thereby enabling us to recover the true ancestry of an individual at a given locus.

\section{Fig 2. Overview of the methods used to simulate genotypes and phenotypes.}

\section{Simulating Phenotypes}

PhenotypeSimulator (25) was used for phenotype simulation, because it allows very fine control over individual effects. It entails a three-step process: (1) simulating phenotype components of interest, (2) scaling the components according to the amount of variance explained and (3) combining the phenotype components for each individual. The phenotype components are further divided into genetic effects and noise effects. Genetic effects are made up of genetic variant effects, population structure, local ancestry and the interaction between variant effects and local ancestry effects. Noise effects are made up of observational noise effects and confounding variable such as sex and age.

Three kinds of phenotypes were simulated for the Nama (Table 1) and the SAC (Table 2) phenotypes with an allelic only (AO) effect, phenotypes with an ancestry plus allelic (APA) effect and phenotypes with an APA and local ancestry adjusted allelic (LAAA) effect. Three replications, each with a different set of 10 causal SNPs of each kind of phenotype were simulated and a replication was simulated with each ancestral population (three for the Nama and five for the SAC) as the source of the local ancestry effect. Therefore, in total 27 phenotypes were simulated for the Nama and 45 phenotypes we simulated for the SAC.

Table 1. Breakdown of the effect sizes that make up the phenotypes simulated for the Nama.

\begin{tabular}{|c|c|c|c|c|c|c|c|}
\hline \multirow[b]{2}{*}{ Phenotype } & \multirow[b]{2}{*}{$\begin{array}{l}\text { Ancestry } \\
\text { of interest }\end{array}$} & \multicolumn{4}{|c|}{ Genetic effects } & \multicolumn{2}{|c|}{ Noise effects } \\
\hline & & Variant & $\begin{array}{c}\text { Local } \\
\text { ancestry }\end{array}$ & Interaction & $\begin{array}{l}\text { Population } \\
\text { structure }\end{array}$ & Observational & Covariates \\
\hline
\end{tabular}




\begin{tabular}{llllllll}
\hline GAO & Nama & 0.12 & 0.00 & 0.00 & 0.48 & 0.16 & 0.24 \\
GAO & GBR & 0.12 & 0.00 & 0.00 & 0.48 & 0.16 & 0.24 \\
GAO & EP & 0.12 & 0.00 & 0.00 & 0.48 & 0.16 & 0.24 \\
APA & Nama & 0.12 & 0.06 & 0.00 & 0.42 & 0.16 & 0.24 \\
APA & GBR & 0.12 & 0.06 & 0.00 & 0.42 & 0.16 & 0.24 \\
APA & EP & 0.12 & 0.06 & 0.00 & 0.42 & 0.16 & 0.24 \\
LAAA & Nama & 0.12 & 0.06 & 0.04 & 0.38 & 0.16 & 0.24 \\
LAAA & GBR & 0.12 & 0.06 & 0.04 & 0.38 & 0.16 & 0.24 \\
LAAA & EP & 0.12 & 0.06 & 0.04 & 0.38 & 0.16 & 0.24 \\
\hline
\end{tabular}

188 Table 2. Breakdown of the effect sizes that make up the phenotypes simulated for the SAC.

\begin{tabular}{|c|c|c|c|c|c|c|c|}
\hline \multirow[b]{2}{*}{ Phenotype } & \multirow[b]{2}{*}{$\begin{array}{l}\text { Ancestry } \\
\text { of interest }\end{array}$} & \multicolumn{4}{|c|}{ Genetic effects } & \multicolumn{2}{|c|}{ Noise effects } \\
\hline & & Variant & $\begin{array}{c}\text { Local } \\
\text { ancestry }\end{array}$ & Interaction & $\begin{array}{l}\text { Population } \\
\text { structure }\end{array}$ & Observational & Covariates \\
\hline GAO & Nama & 0.12 & 0.00 & 0.00 & 0.48 & 0.16 & 0.24 \\
\hline GAO & GBR & 0.12 & 0.00 & 0.00 & 0.48 & 0.16 & 0.24 \\
\hline GAO & MSL & 0.12 & 0.00 & 0.00 & 0.48 & 0.16 & 0.24 \\
\hline GAO & $\mathrm{CHB}$ & 0.00 & 0.00 & 0.00 & 0.60 & 0.16 & 0.24 \\
\hline GAO & GIH & 0.00 & 0.00 & 0.00 & 0.60 & 0.16 & 0.24 \\
\hline APA & Nama & 0.12 & 0.06 & 0.00 & 0.42 & 0.16 & 0.24 \\
\hline APA & GBR & 0.12 & 0.06 & 0.00 & 0.42 & 0.16 & 0.24 \\
\hline APA & MSL & 0.12 & 0.06 & 0.00 & 0.42 & 0.16 & 0.24 \\
\hline APA & $\mathrm{CHB}$ & 0.12 & 0.06 & 0.00 & 0.42 & 0.16 & 0.24 \\
\hline APA & GIH & 0.12 & 0.06 & 0.00 & 0.42 & 0.16 & 0.24 \\
\hline LAAA & Nama & 0.12 & 0.06 & 0.04 & 0.38 & 0.16 & 0.24 \\
\hline LAAA & GBR & 0.12 & 0.06 & 0.04 & 0.38 & 0.16 & 0.24 \\
\hline LAAA & MSL & 0.12 & 0.06 & 0.04 & 0.38 & 0.16 & 0.24 \\
\hline LAAA & CHB & 0.12 & 0.06 & 0.04 & 0.38 & 0.16 & 0.24 \\
\hline LAAA & GIH & 0.12 & 0.06 & 0.04 & 0.38 & 0.16 & 0.24 \\
\hline
\end{tabular}

190 For all phenotypes, genetic effects were assumed to account for $60 \%$ of the total variance.

191 When genetic variant effects were present, they were assumed to account for $20 \%$ of the total

192 genetic effect or $12 \%$ of the total effect, when local ancestry effects are present, they were

193 assumed to account for $10 \%$ of the total genetic effect or $6 \%$ of the total effect and when the

194 interactions between variant effects and local ancestry effects were present, they were assumed

195 to account for $6.67 \%$ of the total genetic effect or $4 \%$ of the total effect. After these effects

196 were accounted for, the proportion of the genetic effect that remains was attributed to 197 population structure and kinship. Observational noise was assumed to be $40 \%$ of the total noise 
effect or $16 \%$ of the total effect and the non-genetic covariates, sex and age, was assumed to account for the remaining $60 \%$ of the noise effect or $24 \%$ of the total effect. These proportions are completely arbitrary and only the genetic variant effects are based on literature - complex diseases, such as type 2 diabetes and systemic lupus erythematosus, have been associated with 4-40 loci with a total heritability explained by variant effects of 1.5-50\% (26).

\section{Ancestry inference}

The simulated Nama and SAC datasets were merged with their respective appropriate source populations using PLINK v2.0 (https://www.cog-genomics.org/plink/2.0/) (27) in order to infer both global and local ancestry. For the Nama, the GBR $(n=1000)$, LWK $(n=1000)$ and Nama $(n=1000)$ were included to represent their contributing ancestral source populations. For the SAC, the GBR $(n=1000)$, MSL $(n=1000)$, Nama $(n=1000)$, GIH $(n=1000)$ and CHB $(\mathrm{n}=1000)$ were included to represent their contributing ancestral source populations. After merging of admixed and source ancestral populations, all minor alleles with a frequency $<0.05$ were excluded from analysis. The final dataset after quality control and data filtering consisted of 387959 autosomal variants and 5000 Nama and 5000 SAC, in addition to 3000 ancestral individuals for the Nama and 5000 ancestral individuals for the SAC. The software RFMix was used to infer global and local ancestry for both the Nama and SAC datasets (28). RFMix is 30X faster than other local ancestry inference software and is accurate in multi-way admixture scenarios $(28,29)$. Default parameters were used, except for the number of generations since admixture, which was set to 13 for the Nama and 16 for the SAC.

\section{GWAS Models}

The following five regression models were tested simultaneously for each simulated phenotype for both the Nama and SAC:

1. The standard model - only allelic effects are considered and no global or local ancestry effects are modeled.

$$
E(Y)=\alpha_{\mathrm{o}}+\alpha_{1} E_{1}+\alpha_{2} E_{2}+\beta X_{\mathrm{x}}
$$
$\boldsymbol{E}(\boldsymbol{Y})$ is the continuous outcome $(Y) \cdot \boldsymbol{E}_{1}$ and $\boldsymbol{E}_{2}$ represent the covariates (age and gender) and $\alpha_{1}$ and $\alpha_{2}$ is the corresponding marginal effect. $X_{\mathrm{x}}$ represents the number 
of reference alleles at the locus under investigation and $\beta$ is the corresponding marginal effect.

2. The global ancestry (GA) model - frequently used in GWAS to account for the possible confounding effect of population structure in the study population.

$$
E(Y)=\alpha_{\mathrm{o}}+\alpha_{1} E_{1}+\alpha_{2} E_{2}+\alpha_{\mathrm{p}} P+\beta X_{\mathrm{x}}
$$

$\boldsymbol{P}$ represents the estimated global ancestry and $\alpha_{\mathrm{p}}$ is the corresponding marginal effect.

3. The local ancestry (LA) model - used in admixture mapping studies to localise potential associations to ancestral blocks. The model is sensitive to frequency disparities across ancestral populations.

$$
E(Y)=\alpha_{\mathrm{o}}+\alpha_{1} E_{1}+\alpha_{2} E_{2}+\alpha_{\mathrm{p}} P+y X^{\mathrm{i}}
$$

$\boldsymbol{X}^{\mathrm{i}}$ represents the number of ancestry alleles (separate model for each contributing ancestry) at the locus under investigation and $y$ is the corresponding marginal effect.

4. The ancestry plus allelic (APA) model - tests for both an allelic association and an association with an ancestry in the phenotype. Therefore jointly testing the associations modelled in model $1+2$.

$$
E(Y)=\alpha_{\mathrm{o}}+\alpha_{1} E_{1}+\alpha_{2} E_{2}+\alpha_{\mathrm{p}} P+\beta X_{\mathrm{x}}+y X^{\mathrm{i}}
$$

5. The local ancestry adjusted allelic (LAAA) model - an extension of the APA model that also includes an interaction term between the allele present at a specific locus and the ancestry.

$$
E(Y)=\alpha_{\mathrm{o}}+\alpha_{1} E_{1}+\alpha_{2} E_{2}+\alpha_{\mathrm{p}} P+\beta X_{\mathrm{x}}+y X^{\mathrm{i}}+\mathrm{\eta}^{\mathrm{i}}{ }_{\mathrm{x}}
$$

$\boldsymbol{X}_{\mathrm{x}}^{\mathrm{i}}$ represents the number of ancestry-specific reference alleles at the locus under investigation and $\eta$ is the corresponding marginal effect.

When global ancestry is included in a given model, the smallest ancestry proportion was excluded as a covariate to avoid complete separation of the data for both the Nama (GBR) and SAC (CHB). Thus, two ancestral components (Nama and LWK) for the Nama and four ancestral components (Nama, MSL, GBR, GIH) for the SAC were included as covariates in association testing, together with gender and age. A total of $5000 \mathrm{Nama}$ and $5000 \mathrm{SAC}$ and 387959 autosomal variants were included in statistical analysis. The $\operatorname{lm}()$ function in $\mathrm{R}$ was used for linear regression association testing. The conventional significance threshold of $5 \times$ $10^{-8}$ for association testing was used. Dosage files were compiled at each locus for the allelic state $(0,1$ or 2 copies of the major allele), for the ancestry $(0,1$ or 2 copies of the ancestry of 
interest) and for the interaction between the allelic state and the ancestry $(0,1$ or 2 copies of the major allele that is from the ancestry of interest).

\section{Results}

267

268

269

270

271

\section{Local ancestry inference accuracy}

We inferred the local ancestry for the simulated Nama and SAC populations, since the true local ancestry will not be available in a GWAS using real data. Therefore, we could also assess the impact of LAI accuracy on the GWAS models in different phenotypic scenarios. An overall $84.90 \%$ accuracy for the Nama (Table 3 ) and an overall $85.40 \%$ accuracy for the SAC was attained. For the ancestral components of the Nama we attained $76.07 \%$ for the LWK, $88.35 \%$ for the GBR and $84.90 \%$ for the Nama (Khoe-San). For the ancestral components of the SAC we attained $83.13 \%$ for the CHB, $89.24 \%$ for the GBR, $84.56 \%$ for the MSL, $84.73 \%$ for the Nama and $85.38 \%$ for the GIH.

Table 3. The ancestral proportions and the accuracy of the inferred local ancestry for the Nama.

\begin{tabular}{ccccc}
\hline & LWK & GBR & Nama & Total \\
\hline Ancestral proportion & 0.100 & 0.150 & 0.750 & 1.000 \\
LAI accuracy & 0.761 & 0.884 & 0.853 & 0.849 \\
\hline
\end{tabular}

Table 4. The ancestral proportions and the accuracy of the inferred local ancestry for the SAC.

\begin{tabular}{ccccccc}
\hline & CHB & GBR & MSL & Nama & GIH & Total \\
\hline Ancestral proportion & 0.100 & 0.175 & 0.250 & 0.350 & 0.125 & 1.000 \\
LAI accuracy & 0.831 & 0.892 & 0.846 & 0.847 & 0.854 & 0.854 \\
\hline
\end{tabular}

\section{Comparison of GWAS models applied to simulated GWAS data for the Nama and the SAC}


We compared the true-positive and the false-positive hits from each GWAS model applied to all of the simulated phenotypes to get an indication of model success rate in the simulated Nama and SAC populations. Three iterations of each phenotype were simulated with different sets of causal SNPs, the GWAS models were run for each iteration and the number of truepositive and false-positive hits (excluding sites in LD with causal SNPs) were averaged to produce the results in Fig 3-6. Across all phenotypes in both populations, whether true (Fig 4 and 6) or inferred (Fig 3 and 5) ancestry was used, the LAAA model achieved the highest or tied for the highest true-positive hit count with the exception of the AO phenotypes in the Nama (Fig 3 and 4) where the standard model sometimes did better. The standard model was tied with the LAAA model for the highest hit count in phenotypes with only an allelic effect (Fig 5-6) in the SAC. In phenotypes that have an ancestry effect in the Nama, either the APA model or the standard model achieved the second highest hit count after the LAAA model (Fig 3-4), however, in the SAC the APA model identified more true-positive hits in all phenotypes (Fig 5 and 6). The LA model did not identify any true-positives in many cases and produced a substantial number of false-positives when there is an ancestry effect present (Fig 3-6). The GA model identified more true-positives than the LA model, but fewer than the standard model. The standard model produced the lowest number of false-positive hits regardless of the phenotype it was applied to, but when there is an ancestry effect present, the standard model never identified more than $6 / 10$ causal variants whereas models such as LAAA sometimes identified $9 / 10$.

All models produced low false-positives for the AO phenotypes relative to the other phenotypes and overall, models with a local ancestry component (APA, LA, LAAA) produced substantially more false-positives than models that only account for allelic effects and global ancestry effects (Fig 3-6). There are more false-positives across phenotypes and models when inferred ancestry was used for the Nama and the SAC instead of the true ancestry (Fig 3-6). For the SAC there are slightly more false-positives for phenotypes with the CHB and GBR as the source of the ancestral effect when using the true ancestry (Fig 6) compared to the inferred ancestry (Fig 5). There appears to be some relationship between the ancestral proportion of the ancestry used for the ancestry effect in the simulated phenotypes and the number of falsepositive hits (Fig 3 and 5). In every phenotype, except the AO phenotypes, the LWK had the lowest number of false-positives in the Nama (Fig 3 and 4) and the CHB had the lowest number of false-positives in the SAC when the inferred ancestry was used (Fig 6). This pattern holds true for the other inferred ancestries (except the GIH) in the SAC phenotypes (Fig 5), however, 
322

323

324

325

326

327

328

329

330

331

332

333

334

335

336

337

338

339

340

341

342

343

344

345

346

347

348

349

350

351

for the other ancestries in the Nama (Fig 3 and 4) a higher ancestral proportion does not result in more false-positives. In the Nama, the use of inferred local ancestry in the APA, LA and GA models (Fig 3) also produced more false-positives in the AO phenotypes than using the true ancestry in these models. Using inferred ancestry over the true ancestry noticeably decreased the number of true-positives detected by the APA, LA and the GA, whereas the LAAA model is less affected by inaccurate inferred local ancestry.

Fig 3. Comparison of true-positive hits (left) and false-positive (right) for the Nama with inferred local ancestry used in GWAS models. The average hits for three runs with different causal SNPs are shown. Simulated phenotypes ae denoted as "phenotype-ancestral source of association", e.g.,LAAA-LWK means the LAAA phenotype with the LWK ancestral component as the ancestral source of association.

\section{Fig 4. Comparison of true-positive hits (left) and false-positive hits (right) for the Nama} with true local ancestry used in GWAS models. The average hits for three runs with different causal SNPs are shown. Simulated phenotypes are denoted as "phenotype-ancestral source of association", e.g.,LAAA-LWK means the LAAA phenotype with the LWK ancestral component as the ancestral source of association.

Fig 5. Comparison of true-positive hits (left) and false-positive hits (right) for the SAC with inferred local ancestry used in GWAS models. The average hits for three runs with different causal SNPs are shown. Simulated phenotypes are denoted as "phenotype-ancestral source of association", e.g.,LAAA-CHB means the LAAA phenotype with the CHB ancestral component as the ancestral source of association.

Fig 6. Comparison of true-positive hits (left) and false-positive hits (right) for the SAC with inferred local ancestry used in GWAS models. The average hits for three runs with different causal SNPs are shown. Simulated phenotypes are denoted as "phenotype-ancestral source of association”, e.g.,LAAA-CHB means the LAAA phenotype with the CHB ancestral components as the ancestral source of association. 


\section{Discussion}

353 Usually, researchers adjust for the possible confounding effect of population structure by

354 considering global ancestry proportions or top principal components in statistical models.

355 Conversely, researchers would conduct admixture mapping, which involves inferring ancestry

356 at each genomic loci along the genome of an admixed individual (local ancestry inference), to

357 increase the power to detect variants with an ancestry effect $(30,31)$. This is sufficient in simple

358 admixture scenarios, such as two-way admixed populations (African-Americans) and three-

359 way admixed populations (Latinos/Hispanics) (1,32,33). Populations from southern Africa,

360 however, can be complex multi-way admixed populations, with up to five contributing

361 ancestries $(34,35)$. Furthermore, the underlying genetic architecture is mostly unknown and can be complex due to various historical events in southern Africa (4). Thus, conventional admixture mapping strategies might result in either increased false-positives or false-negatives for complex multi-way admixed populations. Duan et al. (8) first described LAAA analysis as a robust method for discovering association signals, while producing few false-positives, by comparing the LAAA model to other well-known models used for GWAS in admixed populations (8). Their simulation study, however, was limited to a two-way admixed population. Realising that their findings might not translate to other admixture scenarios, we simulated a three- and five-way admixed population to compare the GA, LA, APA and LAAA models that they tested and added a conventional GWAS model that does not model any local ancestry effects (the standard model). Our main objective is was to discern which statistical model robustly captures the true causal variants in complex multi-way admixed populations without prior knowledge of admixture dynamics.

The GA model is the most widely used model to control for population structure in GWAS, because it has been shown to improve GWAS results when the phenotype exhibits a differential allelic effect across ancestral populations (scenario AO). Therefore, adjusting for only global ancestry proportions or the top principle components should be sufficient to control for the confounding effect of population structure. In phenotypes where there is no ancestry effect, we expected that the GA model would improve upon the standard model, however, this was not the case.

The LA model identified the least amount of true-positive causal variants and sometimes none at all. This makes sense, since only local ancestry is considered in statistical analysis and the 
possible allelic effect is not accounted for. The LA model is more successful in phenotypes where the ancestral component that causes the ancestry effect is small, which is expected, since the effect is concentrated in fewer SNPs. We only observed this effect in the five-way admixed population and not in the three-way admixed population (Figure 4 and 6). This effect could be due to chance, since causal SNPs were drawn randomly from the dataset without any regard for the local ancestry of the SNP. Our simulations would have to be adjusted to ensure that all of the causal SNPs are found in or outside of the contributing ancestry's ancestral blocks and many more iterations would have to be done to test this. Any success in identifying true causal variants with this method is negated by the large number of false-positive hits that are produced. The false-positive hits produced by this model are generally organised into admixture peaks where all the SNPs that share an ancestral block with the causal variant will have a strong association with the phenotype. This approach only indicates the ancestral block associated with the disease trait and, therefore, requires more information to identify which variant is responsible for the association perceived in the admixture peak. Furthermore, when the simulated local ancestry effects are sufficiently large, over inflation of test statistics could occur.

The APA model is expected to perform better than the first two models when there is both an allelic and ancestry effect, because both of these effects are modelled at each genomic locus. When testing genetic markers that are proxies for a disease causal loci, the differential LD within admixed populations can result in heterogeneity of effect estimates by local ancestry (36). Therefore, additional adjustments beyond global ancestry are required to increase power when the admixture-induced LD is in the opposite direction as the LD in the ancestral population $(8,36)$. This agrees with what we observe from our results where the GA model identified fewer true-positive hits in comparison to the APA and LAAA models when the simulated phenotypes displayed a local ancestry effect. The APA model is also more readily affected by LAI accuracy than the LAAA model. Hence, the APA model could be more prone to false-negatives and false-positives. The APA model might also be insufficient for certain admixture scenarios, such as scenarios where admixture induced LD blocks mask the allelic effect of the causal SNP when the tagging SNP is located in a different ancestral LD block to the causal SNP.

The LAAA model outperforms the other statistical models by either achieving the most truepositive hits or achieving the second highest hit count. Most importantly, the LAAA is 
particularly robust in the presence of genetic effect heterogeneity. Interestingly, Duan et al. (8) and Liu et al. (36) both recommend to first identify admixture peaks and then to use the interaction term as a secondary follow-up test to elucidate the source of the association obtained in the admixture peak. However, our results demonstrate the possible true causal variants that might be missed when not including the interaction term from the beginning in complex multiway admixed populations. Importantly, the LAAA model was robust against inaccurate local ancestry inference ( 0.849 for the Nama and 0.854 for the SAC) with only minor changes in the number of true-positives between GWAS performed with inferred ancestry versus true

We assumed independence of ancestry-specific effects across chromosomes. Therefore, the effect of other ancestral blocks (besides the ancestral blocks in which the causal SNPs are found) on a given phenotype was not simulated. This is important to note when considering our results in terms of real data, since this omission can have an impact on elucidating the true causal SNPs. Future studies should consider including all five ancestries in a linear mixed model to account for cases where traits are affected by the interaction between multiple ancestral blocks and their interaction with the causal SNPs. Another unexpected observation in the results, is the large number of false-positives for both the APA and LAAA models compared to the other three models (Standard, GA and LA). This could be due to the simulated local ancestry effect size being too large, causing associations of whole ancestral blocks with the trait of interest. This study was limited by only conducting three replications of the simulated genotypes and phenotypes. Ideally, we would perform a few thousand replications to allow us to calculate the power of each model to identify causal SNPs. However, we were limited by the computational resources required for such an experiment. Alternatively, we could have reduced the number of SNPs used in the analysis and we could have simulated a single population instead of two. The impact of changes of the mutation rate and recombination rate on the statistical models ability to capture true variants could also be tested in the future.

446 The composition of the diverse ancestral haplotype LD blocks depends on the time since 447 admixture, as well as the admixture proportions. Therefore, future work could also include more complex admixture scenarios.

In conclusion, investigating admixed individuals can be advantageous, due to diverse patterns of LD across continental populations. Nevertheless, the differential allele frequencies and LD patterns that exist between the ancestries should be incorporated correctly for true causal 
453

454

455

456

457

458

459

460

461

462

463

464

465

466

467

468

469

470

471

472

473

474

475

476

477

478

479

480

481

variants to be identified. The findings of this study show that jointly testing an allele effect, ancestry effect, and allele effect heterogeneity across ancestry (LAAA) in a regression model guards against missing genuine associations from any of the three sources. However, it is evident from the large number of false positives detected by some models, that the ancestry effect size we assumed across our simulated phenotypes was too large. Therefore, we were unable to fully assess the suitability of the LAAA model in a complex multi-way admixture scenario. Considering the extent to which the various models we tested differed in their results and considering that the source of a given association is unknown, we recommend that researchers use multiple GWAS models when analysing populations with complex ancestry. Specifically when the population under study exhibits extensive genetic diversity and complicated LD patterns, which are often observed for southern African populations.

\section{Acknowledgements}

GvE contributed towards the introduction, results, methods, figures and discussion, especially as it pertains to simulating genotypes and phenotypes. YS contributed to the introduction, results, methods, and discussion, especially as it pertains to GWAS models. All authors contributed to design of the study and the writing and proofreading of the final manuscript.

\section{Data availability Statement}

The data analyzed in this study is subject to the following licenses/restrictions: No new genetic data was generated for this study however, summary statistics for the quality and accuracy assessment of the genetic data will be made available to researchers who meet the criteria for access after application to the Health Research Ethics Committee of Stellenbosch University. Requests to access these datasets should be directed to MM, marlom@sun.ac.za

\section{References}

Akenroye, A.T., Brunetti, T., Romero, K., Daya, M., Kanchan, K., Shankar, G., Chavan, S., Preethi Boorgula, M., et al. 2021. Genome-wide association study of asthma, total IgE, and lung function in a cohort of Peruvian children. The Journal of Allergy and Clinical Immunology. (March, 10). 
Atkinson, E.G., Dalvie, S., Pichkar, Y., Kalungi, A., Majara, L., Stevenson, A., Abebe, T., Akena, D., et al. 2021. Genetic structure correlates with ethnolinguistic diversity in eastern and southern Africa. BioRxiv. (May, 19).

Baran, Y., Pasaniuc, B., Sankararaman, S., Torgerson, D.G., Gignoux, C., Eng, C., RodriguezCintron, W., Chapela, R., et al. 2012. Fast and accurate inference of local ancestry in Latino populations. Bioinformatics. 28(10):1359-1367.

Barbieri, C., Vicente, M., Rocha, J., Mpoloka, S.W., Stoneking, M. \& Pakendorf, B. 2013. Ancient substructure in early mtDNA lineages of southern Africa. American Journal of Human Genetics. 92(2):285-292.

Barbieri, C., Hübner, A., Macholdt, E., Ni, S., Lippold, S., Schröder, R., Mpoloka, S.W., Purps, J., et al. 2016. Refining the Y chromosome phylogeny with southern African sequences. Human Genetics. 135(5):541-553.

Brown, L.A., Sofer, T., Stilp, A.M., Baier, L.J., Kramer, H.J., Masindova, I., Levy, D., Hanson, R.L., et al. 2017. Admixture Mapping Identifies an Amerindian Ancestry Locus Associated with Albuminuria in Hispanics in the United States. Journal of the American Society of Nephrology. 28(7):2211-2220.

Chen, J., Spracklen, C.N., Marenne, G., Varshney, A., Corbin, L.J., Luan, J., Willems, S.M., $\mathrm{Wu}, \mathrm{Y}$., et al. 2021. The trans-ancestral genomic architecture of glycemic traits. Nature Genetics. 53(6):840-860.

Chimusa, E.R., Zaitlen, N., Daya, M., Möller, M., van Helden, P.D., Mulder, N.J., Price, A.L. \& Hoal, E.G. 2014. Genome-wide association study of ancestry-specific TB risk in the South African Coloured population. Human Molecular Genetics. 23(3):796-809.

Daya, M., van der Merwe, L., van Helden, P.D., Möller, M. \& Hoal, E.G. 2014. The role of ancestry in TB susceptibility of an admixed South African population. Tuberculosis. 94(4):413-420.

Duan, Q., Xu, Z., Raffield, L.M., Chang, S., Wu, D., Lange, E.M., Reiner, A.P. \& Li, Y. 2018. A robust and powerful two-step testing procedure for local ancestry adjusted allelic association analysis in admixed populations. Genetic Epidemiology. 42(3):288-302.

Gronau, I., Hubisz, M.J., Gulko, B., Danko, C.G. \& Siepel, A. 2011. Bayesian inference of ancient human demography from individual genome sequences. Nature Genetics. 43(10):1031-1034. 
Henn, B.M., Gignoux, C., Lin, A.A., Oefner, P.J., Shen, P., Scozzari, R., Cruciani, F., Tishkoff, S.A., et al. 2008. Y-chromosomal evidence of a pastoralist migration through Tanzania to southern Africa. Proceedings of the National Academy of Sciences of the United States of America. 105(31):10693-10698.

Henn, B.M., Gignoux, C.R., Jobin, M., Granka, J.M., Macpherson, J.M., Kidd, J.M., Rodríguez-Botigué, L., Ramachandran, S., et al. 2011. Hunter-gatherer genomic diversity suggests a southern African origin for modern humans. Proceedings of the National Academy of Sciences of the United States of America. 108(13):5154-5162.

Horimoto, A.R.V.R., Xue, D., Thornton, T.A. \& Blue, E.E. 2021. Admixture mapping reveals the association between Native American ancestry at 3q13.11 and reduced risk of Alzheimer's disease in Caribbean Hispanics. Alzheimer's research \& therapy. 13(1):122.

Kelleher, J., Etheridge, A.M. \& McVean, G. 2016. Efficient coalescent simulation and genealogical analysis for large sample sizes. PLoS Computational Biology. 12(5):e1004842.

Kelleher, J., Thornton, K.R., Ashander, J. \& Ralph, P.L. 2018. Efficient pedigree recording for fast population genetics simulation. PLoS Computational Biology. 14(11):e1006581.

Liu, J., Lewinger, J.P., Gilliland, F.D., Gauderman, W.J. \& Conti, D.V. 2013. Confounding and heterogeneity in genetic association studies with admixed populations. American Journal of Epidemiology. 177(4):351-360.

Manolio, T.A., Collins, F.S., Cox, N.J., Goldstein, D.B., Hindorff, L.A., Hunter, D.J., McCarthy, M.I., Ramos, E.M., et al. 2009. Finding the missing heritability of complex diseases. Nature. 461(7265):747-753.

Maples, B.K., Gravel, S., Kenny, E.E. \& Bustamante, C.D. 2013. RFMix: a discriminative modeling approach for rapid and robust local-ancestry inference. American Journal of Human Genetics. 93(2):278-288.

Meyer, H.V. \& Birney, E. 2018. PhenotypeSimulator: A comprehensive framework for simulating multi-trait, multi-locus genotype to phenotype relationships. Bioinformatics. 34(17):2951-2956.

Pickrell, J.K., Patterson, N., Barbieri, C., Berthold, F., Gerlach, L., Güldemann, T., Kure, B., Mpoloka, S.W., et al. 2012. The genetic prehistory of southern Africa. Nature Communications. 3:1143. 
Price, A.L., Tandon, A., Patterson, N., Barnes, K.C., Rafaels, N., Ruczinski, I., Beaty, T.H., Mathias, R., et al. 2009. Sensitive detection of chromosomal segments of distinct ancestry in admixed populations. PLoS Genetics. 5(6):e1000519.

Purcell, S., Neale, B., Todd-Brown, K., Thomas, L., Ferreira, M.A.R., Bender, D., Maller, J., Sklar, P., et al. 2007. PLINK: a tool set for whole-genome association and populationbased linkage analyses. American Journal of Human Genetics. 81(3):559-575.

Ragsdale, A., Weaver, T., Henn, B. \& Gravel, S. 2021. A weakly structured stem for human origins in Africa. in The third Cold Spring Harbor conference on Probabilistic Modeling in Genomics: Cold Spring Harbor Laboratory.

Sajuthi, S.P., Sharma, N.K., Chou, J.W., Palmer, N.D., McWilliams, D.R., Beal, J., Comeau, M.E., Ma, L., et al. 2016. Mapping adipose and muscle tissue expression quantitative trait loci in African Americans to identify genes for type 2 diabetes and obesity. Human Genetics. 135(8):869-880.

Schlebusch, C.M., Skoglund, P., Sjödin, P., Gattepaille, L.M., Hernandez, D., Jay, F., Li, S., De Jongh, M., et al. 2012. Genomic variation in seven Khoe-San groups reveals adaptation and complex African history. Science. 338(6105):374-379.

Schubert, R., Andaleon, A. \& Wheeler, H.E. 2020. Comparing local ancestry inference models in populations of two- and three-way admixture. PeerJ. 8:e10090.

Sengupta, D., Choudhury, A., Fortes-Lima, C., Aron, S., Whitelaw, G., Bostoen, K., Gunnink, H., Chousou-Polydouri, N., et al. 2021. Genetic substructure and complex demographic history of South African Bantu speakers. Nature Communications. 12(1):2080.

Shriner, D. 2017. Overview of admixture mapping. Current Protocols in Human Genetics. 94:1.23.1-1.23.8.

Suarez-Pajes, E., Díaz-de Usera, A., Marcelino-Rodríguez, I., Guillen-Guio, B. \& Flores, C. 2021. Genetic ancestry inference and its application for the genetic mapping of human diseases. International Journal of Molecular Sciences. 22(13).

Uren, C., Kim, M., Martin, A.R., Bobo, D., Gignoux, C.R., van Helden, P.D., Möller, M., Hoal, E.G., et al. 2016. Fine-Scale Human Population Structure in Southern Africa Reflects Ecogeographic Boundaries. Genetics. 204(1):303-314.

Uren, C., Möller, M., van Helden, P.D., Henn, B.M. \& Hoal, E.G. 2017. Population structure and infectious disease risk in southern Africa. Molecular Genetics and Genomics. 
576 Uren, C., Hoal, E.G. \& Möller, M. 2020. Putting RFMix and ADMIXTURE to the test in a complex admixed population. BMC Genetics. 21(1):40.

578 Visscher, P.M., Wray, N.R., Zhang, Q., Sklar, P., McCarthy, M.I., Brown, M.A. \& Yang, J. 579 2017. 10 years of GWAS discovery: biology, function, and translation. American Journal of Human Genetics. 101(1):5-22.

581 Wang, C., Zhan, X., Bragg-Gresham, J., Kang, H.M., Stambolian, D., Chew, E.Y., Branham, K.E., Heckenlively, J., et al. 2014. Ancestry estimation and control of population stratification for sequence-based association studies. Nature Genetics. 46(4):409-415.

584 de Wit, E., Delport, W., Rugamika, C.E., Meintjes, A., Möller, M., van Helden, P.D., Seoighe, 585 C. \& Hoal, E.G. 2010. Genome-wide analysis of the structure of the South African Coloured Population in the Western Cape. Human Genetics. 128(2):145-153. 

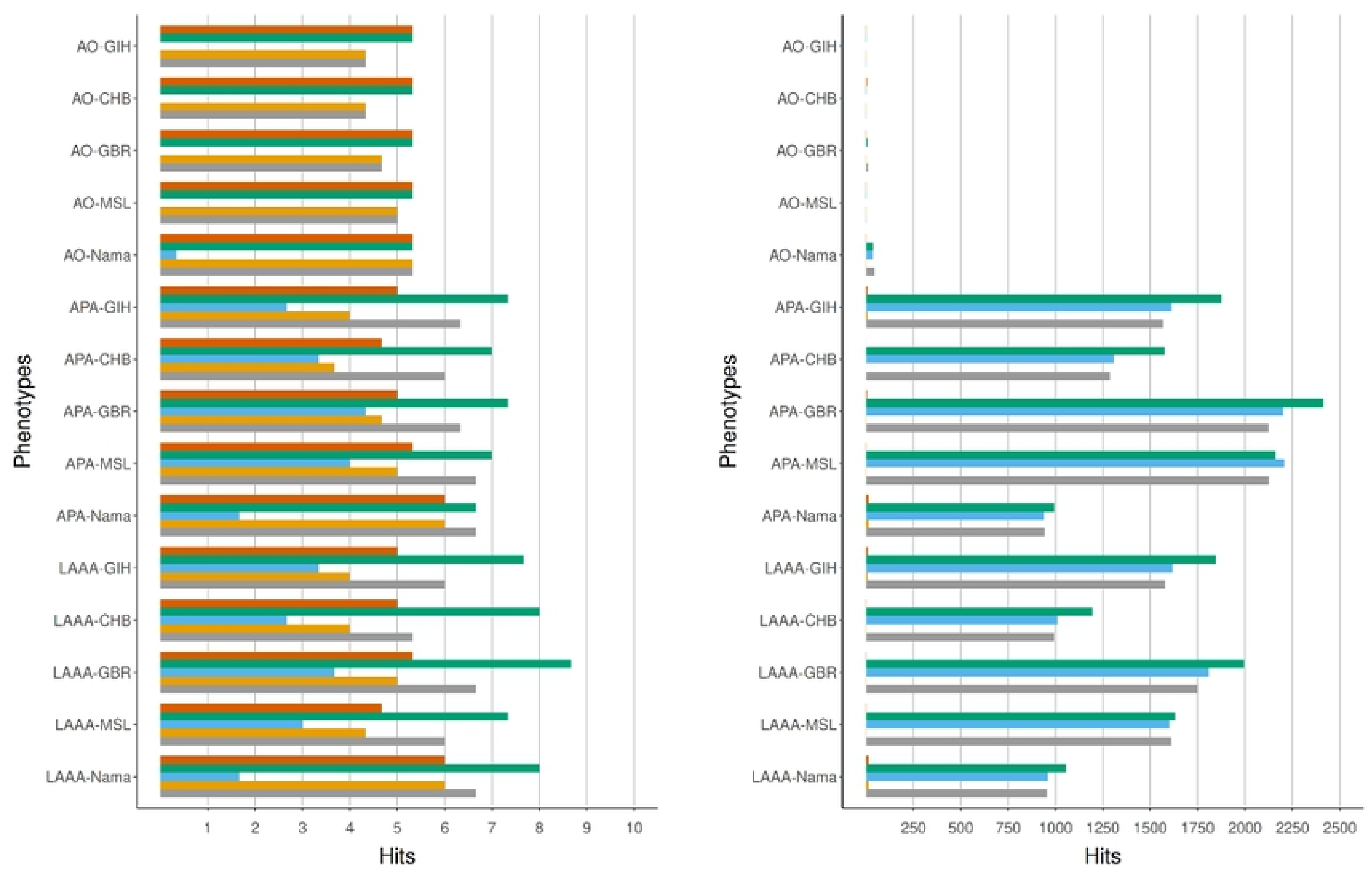

GWAS model $\square$ APA $\square$ GA $\square$ LA $\square$ LAAA $\square$ Standard

Figure6 

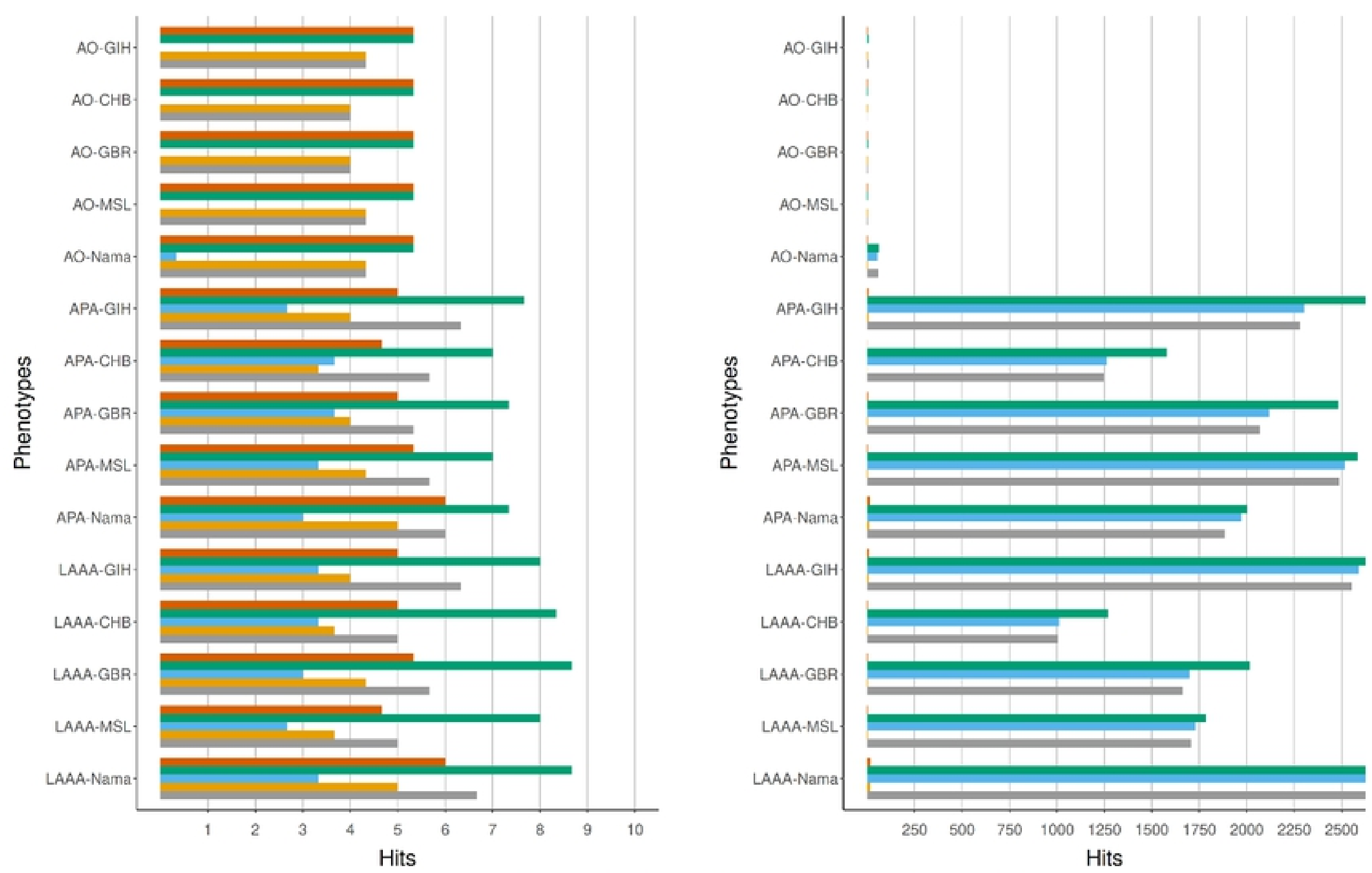

GWAS model $\square$ APA $\square$ GA $\square$ LA $\square$ LAAA $\square$ Standard

Figure5 

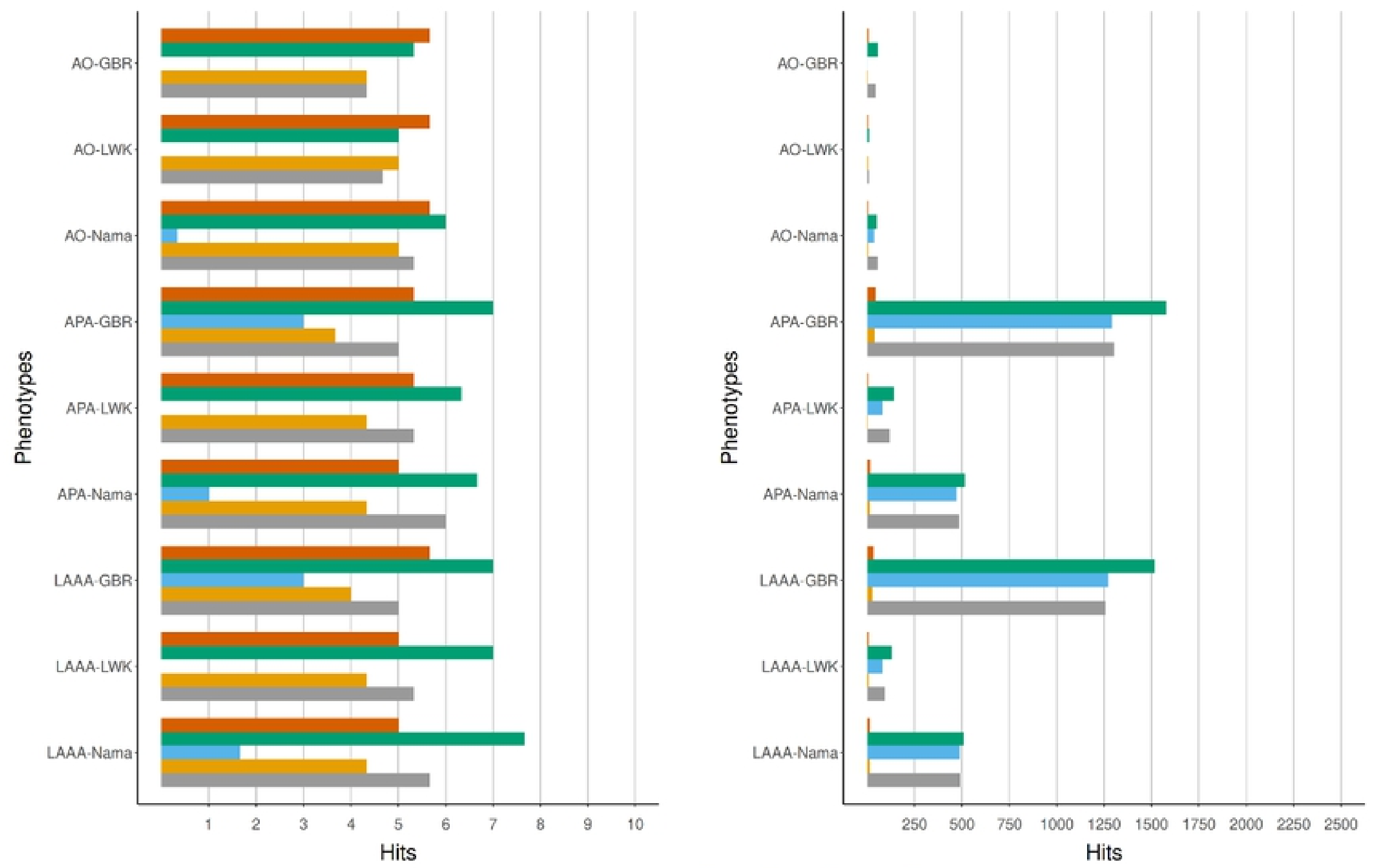

GWAS model $\square$ APA $\square$ GA $\square$ LA $\square$ LAAA $\square$ Standard

Figure4 


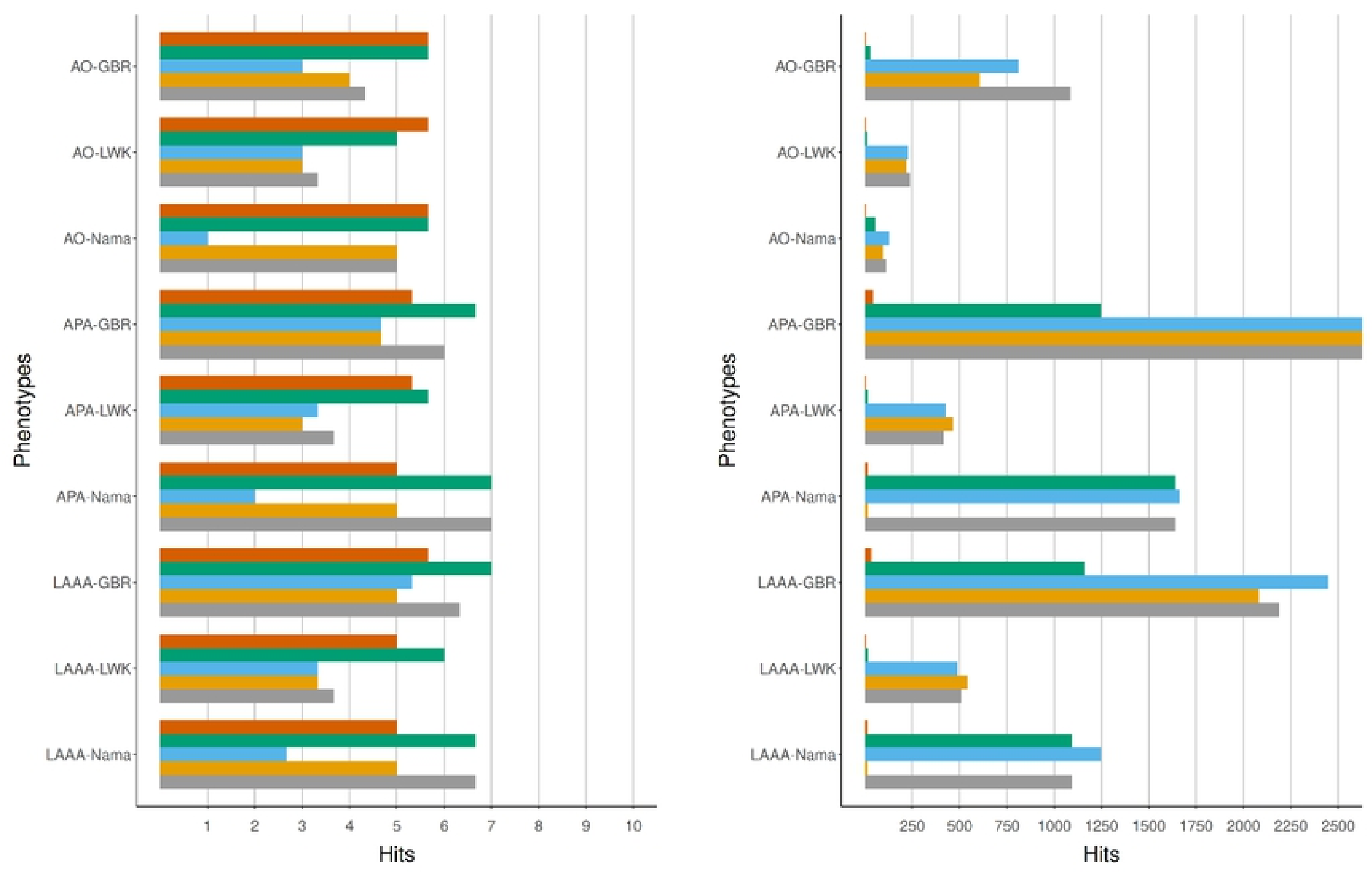

GWAS model $\square$ APA $\square$ GA $\square$ LA $\square$ LAAA $\square$ Standard

Figure3 


\section{Simulations}

\section{Simulating genotypes with msprime}

\begin{tabular}{|c|c|c|}
$\begin{array}{c}\text { Describe the demographic } \\
\text { history using a Demes } \\
\text { Specification }\end{array}$ & $\longrightarrow$ & $\begin{array}{c}\text { Simulate a tree sequence } \\
\text { and mutations while } \\
\text { tracking migrations } \\
\text { Use information on } \\
\text { migrations to retrieve the } \\
\text { true local ancestry of } \\
\text { individuals }\end{array}$ \\
\hline
\end{tabular}

\section{Simulating phenotypes with PhenoypeSimulator}

Simulate components of

interest: genetic variant effects, population structure, observational noise effects, etc.

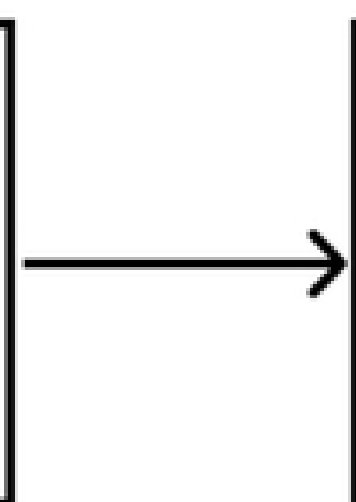

Scale components according to the amount of variance explained by each component
Combine scaled components into final phenotype

\section{Figure2}

